\title{
Mudança no comportamento sexual dos jovens e aumento da vulnerabilidade às infecções sexualmente transmissíveis: uma revisão narrativa de literatura
}

Changing sexual behavior among young people and increasing vulnerability to sexually transmitted infections: a narrative literature review

Cambiar el comportamiento sexual entre los jóvenes y aumentar la vulnerabilidad a las infecciones de transmisión sexual: una revisión narrativa de la literatura

Luana Damaceno Miranda ORCID: https://orcid.org/0000-0003-4013-2414 Centro Universitário de Patos de Minas, Brasil E-mail: luanad.m@hotmail.com

Ana Flávia Braz de Morais ORCID: https://orcid.org/0000-0002-6040-2808 Centro Universitário de Patos de Minas, Brasil E-mail: anabraz@unipam.edu.br

Ana Paula Gomes Tavares ORCID: https://orcid.org/0000-0002-2019-5250 Universidade de Uberaba, Brasil E-mail: anatavares2625@gmail.com

Bárbara Queiroz de Figueiredo ORCID: https://orcid.org/0000-0003-1630-4597 Centro Universitário de Patos de Minas, Brasil

E-mail: barbarafigueiredo@unipam.edu.br

Eduarda Canedo Nogueira

ORCID: https://orcid.org/0000-0002-3654-9228 Centro Universitário de Patos de Minas, Brasil E-mail: eduardacanedo@hotmail.com

Gardênia Silva Amorim ORCID: https://orcid.org/0000-0001-7888-8562 Centro Universitário de Atenas, Brasil

E-mail: gardenia.sa@hotmail.com

Nicolly Skarlet Souto Oliveira ORCID: https://orcid.org/0000-0003-3304-9946 Centro Universitário de Patos de Minas, Brasil

E-mail: nicollyskarlet@gmail.com

Rúbia Carla Oliveira

ORCID: https://orcid.org/0000-0002-7162-3497 Centro Universitário de Patos de Minas, Brasil E-mail: rubiacoliveira@unipam.edu.br

\begin{abstract}
Resumo
Introdução: a adolescência vem sendo encarada no cenário brasileiro e mundial como um período de intensas e abruptas mudanças físicas, emocionais e principalmente comportamentais. Com o advento da AIDS e o emergir das infecções sexualmente transmissíveis (ISTs) - causas importantes de morbimortalidade no Brasil -, o sistema de saúde ampliou a visão dos fatores de risco associados às doenças transmissíveis e trouxe à discussão a questão da vulnerabilidade e sexualidade. Objetivo: explanar acerca das mudanças de comportamento sexual entre os jovens, mesmo perante maior debate sobre o tema, e a incidência de ISTs, que continua alta. Metodologia: trata-se de uma revisão narrativa de literatura. A pesquisa foi realizada através do acesso online nas bases de dados National Library of Medicine (PubMed MEDLINE), Scientific Electronic Library Online (Scielo), Cochrane Database of Systematic Reviews (CDSR), Google Scholar, Biblioteca Virtual em Saúde (BVS) e EBSCO Information Services, no mês de outubro de 2021. Resultados: há uma mudança significativa de comportamento dos jovens quanto à saúde sexual e ao modo como se relacionam com seus parceiros. Além disso, nota-se que a geração atual se expõe mais, haja vista que, devido ao advento da internet e, posteriormente, dos aplicativos de relacionamento, houve uma revolução sobre as formas de interação interpessoal, trazendo muitas facilidades, em especial para a população LGBTQIA+, que encontrou nestes instrumentos virtuais um espaço seguro de contato afetivo e sexual. Considerações finais: é importante que jovens sejam orientados em relação às suas práticas sexuais. Para além do estudo sobre anatomia, a educação sexual proporciona o acesso a informações sobre diferentes aspectos da sexualidade. A partir dela, pessoas
\end{abstract}


se tornam aptas a exercerem seus desejos sexuais e reprodutivos, estando informados quanto à sua saúde, haja vista que a sexualidade é costurada por diversas dimensões, como a biológica, social e cultural.

Palavras-chave: Sexualidade; Adolescentes; ISTs; Educação sexual.

\begin{abstract}
Introduction: adolescence has been seen in the Brazilian and worldwide scenario as a period of intense and abrupt physical, emotional and mainly behavioral changes. With the advent of AIDS and the emergence of sexually transmitted infections (STIs) - important causes of morbidity and mortality in Brazil - the health system expanded the view of risk factors associated with transmissible diseases and brought to the discussion the issue of vulnerability and sexuality. Objective: to explain about changes in sexual behavior among young people, even in the face of greater debate on the subject, and the incidence of STIs, which remains high. Methodology: this is a narrative literature review. The search was carried out through online access in the National Library of Medicine (PubMed MEDLINE), Scientific Electronic Library Online (Scielo), Cochrane Database of Systematic Reviews (CDSR), Google Scholar, Virtual Health Library (BVS) and EBSCO databases Information Services, October 2021. Results: there is a significant change in the behavior of young people regarding sexual health and the way they relate to their partners. In addition, it is noted that the current generation is more exposed, given that, due to the advent of the internet and, later, relationship applications, there was a revolution in the forms of interpersonal interaction, bringing many facilities, especially for the LGBTQIA+ population, who found in these virtual instruments a safe space for affective and sexual contact. Final considerations: it is important that young people are guided in relation to their sexual practices. In addition to the study of anatomy, sex education provides access to information about different aspects of sexuality. From it, people become able to exercise their sexual and reproductive desires, being informed about their health, given that sexuality is linked by different dimensions, such as biological, social and cultural.
\end{abstract}

Keywords: Sexuality; Teenagers; ISTs; Sex education.

\title{
Resumen
}

Introducción: la adolescencia ha sido vista en el escenario brasileño y mundial como un período de intensos y abruptos cambios físicos, emocionales y principalmente conductuales. Con el advenimiento del sida y la aparición de las infecciones de transmisión sexual (ITS), importantes causas de morbilidad y mortalidad en Brasil, el sistema de salud amplió la visión de los factores de riesgo asociados a las enfermedades transmisibles y llevó a la discusión el tema de la vulnerabilidad y la sexualidad. Objetivo: explicar los cambios en el comportamiento sexual de los jóvenes, incluso ante un mayor debate sobre el tema, y la incidencia de ITS, que se mantiene alta. Metodología: se trata de una revisión narrativa de la literatura. La búsqueda se realizó a través del acceso en línea en la Biblioteca Nacional de Medicina (PubMed MEDLINE), Scientific Electronic Library Online (Scielo), Cochrane Database of Systematic Reviews (CDSR), Google Scholar, Virtual Health Library (BVS) y bases de datos EBSCO Information Services, Octubre de 2021. Resultados: hay un cambio significativo en el comportamiento de los jóvenes con respecto a la salud sexual y la forma en que se relacionan con sus parejas. Además, se observa que la generación actual está más expuesta, dado que, debido al advenimiento de internet y, posteriormente, las aplicaciones de relación, hubo una revolución en las formas de interacción interpersonal, trayendo muchas facilidades, especialmente para los LGBTQIA +. población, quienes encontraron en estos instrumentos virtuales un espacio seguro para el contacto afectivo y sexual. Consideraciones finales: es importante que los jóvenes sean guiados en relación a sus prácticas sexuales. Además del estudio de la anatomía, la educación sexual brinda acceso a información sobre diferentes aspectos de la sexualidad. A partir de ella, las personas se vuelven capaces de ejercitar sus deseos sexuales y reproductivos, informándose sobre su salud, dado que la sexualidad está ligada por diferentes dimensiones, como la biológica, la social y la cultural.

Palabras clave: Sexualidad; Adolescentes; IST; Educación sexual.

\section{Introdução}

A adolescência vem sendo encarada no cenário brasileiro e mundial como um período de intensas e abruptas mudanças físicas, emocionais e principalmente comportamentais. Uma fase de sonhos, pensamentos mágicos sobre a realidade, que conduz à ideia de uma proteção natural a agressões e riscos externos. Com o advento da AIDS e o emergir das infecções sexualmente transmissíveis (ISTs) - causas importantes de morbimortalidade no Brasil -, o sistema de saúde ampliou a visão dos fatores de risco associados às doenças transmissíveis e trouxe à discussão a questão da vulnerabilidade e sexualidade. Por isso, as medidas de enfrentamento dos problemas emergentes da adolescência ultrapassaram as fronteiras da saúde e alcançaram proporções intersetoriais importantes. Assim, com o avanço das discussões políticas a respeito dos direitos sexuais e reprodutivos, em que o movimento feminista teve forte participação, ampliaram-se as discussões acerca do gênero e da sexualidade para além do caráter biológico, e há décadas, a Organização Mundial de Saúde (OMS) e a Associação Mundial 
para a Saúde Sexual (WAS) têm enfatizado o aspecto multidimensional da saúde sexual e sua relação com direitos e justiça social (Nogueira et al., 2020).

A atenção em saúde sexual e em saúde reprodutiva é uma das áreas de atuação prioritárias da Atenção Básica à Saúde, devendo ser ofertada observando-se como princípio o respeito aos direitos sexuais e aos direitos reprodutivos. Neste contexto, cabe ressaltar que a saúde sexual e reprodutiva encontram-se incluídas entre os oito Objetivos de Desenvolvimento do Milênio definidos na Conferência do Milênio, realizada pela Organização das Nações Unidas (ONU) desde setembro de 2000, através de quatro pontos: a promoção da igualdade entre os sexos e a autonomia das mulheres; a melhoria da saúde materna; o combate ao HIV malária e outras doenças; e a redução da mortalidade infantil (Pinheiro et al., 2017).

Outrossim, os estudos da sexualidade e da saúde sexual, além de seu ensino nos sistemas de educação, historicamente são embasados em visões normativas marcadas, principalmente, por aspectos biológicos cujos discursos estão ancorados nos pressupostos da moral religiosa e reforçados pelo caráter higiênico das estratégias de saúde pública. Tal adequação emana de uma construção social heteronormativa que reprime e disciplina os corpos, conforme estudaram Freud, no início do século XX e Foucault, na segunda metade do século XX. Essa construção social designa papéis sociais e performances a serem seguidas conforme o sexo desde a tenra idade no seio familiar. De modo geral, essas concepções são incorporadas e internalizadas por crianças e adolescentes, refletindo-se em seus comportamentos, principalmente no que diz respeito à sexualidade, às relações pessoais, bem como no acesso a informações e serviços de saúde sexual e saúde reprodutiva (Moreira et al., 2021).

Em 1989, o Ministério da Saúde criou o Programa de Saúde do Adolescente (PROSAD), a partir de deliberações da $42^{\circ}$ Assembleia Mundial de Saúde. Os pressupostos presentes nessa proposta norteiam-se nos princípios do SUS, estando fundamentados na promoção da saúde e na identificação e tratamento de agravos sociais. Nesse sentido, dentre as problemáticas prioritárias a serem abordadas com esse público, destacam-se a gravidez na adolescência, as doenças sexualmente transmissíveis e o uso abusivo de álcool e outras drogas. É importante ressaltar que o PROSAD fundamentou- se na busca pelo atendimento integral do adolescente, de modo que os dispositivos da atenção básica assumissem um papel importante na implementação dessa política, uma vez que se encontram localizados no contexto em que o adolescente está inserido. Já em 1996, foi aprovada a terceira e mais recente Lei de Diretrizes e Bases da Educação Nacional (LDB), que deu origem aos Parâmetros Curriculares Nacionais (PCN) publicados em 1998, cujo objetivo da orientação sexual é contribuir para que os alunos possam desenvolver e exercer sua sexualidade com prazer, saúde e responsabilidade (Almeida et al., 2017).

Para isso, deveria ser trabalhada transversalmente, perpassando todas as disciplinas, em consonância com uma visão ampla de sexualidade, incluindo seu caráter cultural, social e histórico. Assim, o Estado trouxe para a escola a responsabilidade de desenvolver uma ação crítica, reflexiva e educativa para a promoção da saúde de crianças e adolescentes, dividindo essa tarefa com a família. Mesmo o tema não sendo novo no contexto escolar, ganhou força, maior visibilidade e inserção nos currículos escolares devido ao contexto histórico associado à dimensão epidemiológica e às mudanças no padrão de comportamento sexual da sociedade brasileira. No setor saúde, a educação sexual ganhou espaço a partir das políticas públicas de promoção norteadas pela Lei Orgânica da Saúde (lei n. 8.080/90). Visto isso, a educação sexual como estratégia de saúde pública pode interferir - e contribuir diretamente - na redução dos indicadores de morbimortalidade que atingem jovens e adolescentes, como as taxas de incidência de infecções sexualmente transmissíveis (ISTs) (Araújo et al., 2019). No entanto, nota-se que, ao observar as mudanças de comportamento sexual entre os jovens, mesmo perante maior debate sobre o tema, a incidência de ISTs continua alta. Sob essa perspectiva, o objetivo deste estudo é explanar acerca dessa controvérsia, apontando possíveis hipóteses e reflexões. 


\section{Metodologia}

Trata-se de uma pesquisa descritiva do tipo revisão narrativa da literatura, que buscou explanar acerca das mudanças de comportamento sexual entre os jovens, mesmo perante maior debate sobre o tema, e a incidência de ISTs, que continua alta. A pesquisa foi realizada através do acesso online nas bases de dados National Library of Medicine (PubMed MEDLINE), Scientific Electronic Library Online (Scielo), Cochrane Database of Systematic Reviews (CDSR), Google Scholar, Biblioteca Virtual em Saúde (BVS) e EBSCO Information Services, no mês de outubro de 2021. Para a busca das obras foram utilizadas as palavras-chaves presentes nos descritores em Ciências da Saúde (DeCS): em português: "IST", “AIDS”, “jovens", “epidemiologia", “educação sexual”, "incidência", "Brasil”. Como critérios de inclusão, foram considerados artigos originais, que abordassem o tema pesquisado e permitissem acesso integral ao conteúdo do estudo, publicados no período de 2009 a 2021, em português. O critério de exclusão foi imposto naqueles trabalhos que não estavam em português, que não tinham passado por processo de Peer-View e que não abordassem o tema. A estratégia de seleção dos artigos seguiu as seguintes etapas: busca nas bases de dados selecionadas; leitura dos títulos de todos os artigos encontrados e exclusão daqueles que não abordavam o assunto; leitura crítica dos resumos dos artigos e leitura na íntegra dos artigos selecionados nas etapas anteriores. Assim, totalizaram-se 16 artigos científicos para a revisão narrativa da literatura, com os descritores apresentados acima, dos últimos doze anos e em língua portuguesa.

\section{Resultados e Discussão}

De acordo com o painel de indicadores epidemiológicos de sífilis, do Ministério da Saúde, de 2010 a 2016, todas as regiões brasileiras apresentaram grande aumento dos casos de infecção. Em 2016, foram 37.884 registros de pessoas com o vírus HIV. Novos dados do Boletim Epidemiológico HIV/Aids, do Ministério da Saúde, revelam uma queda em 2017: 16.371 pessoas diagnosticadas. Apesar da redução de notificações em 2017, é necessário estar atento ao comportamento sexual de jovens, e há uma grande preocupação com as novas gerações. A tendência das últimas décadas de diminuição destes índices a cada geração se reverteu. Sobre os motivos que podem ter levado a essa situação, ressalta-se que há uma mudança significativa de comportamento dos jovens quanto à saúde sexual e ao modo como se relacionam com seus parceiros. Entre a faixa etária de 18 a 24 anos, 34\% dos diagnosticados não realizam tratamento com antirretrovirais, segundo o Relatório de Monitoramento Clínico do HIV, do Ministério da Saúde. Também há negligência quanto ao uso do preservativo, o método mais indicado para a prevenção de ISTs. De acordo com uma pesquisa de 2015, do Instituto Brasileiro de Geografia e Estatística (IBGE), dos $27,5 \%$ dos estudantes do nono ano do ensino fundamental sexualmente ativos, apenas 66,2\% tinham usado preservativo na última relação sexual (Tabnet, 2017).

Ademais, é possível que o vírus HIV e a AIDS (síndrome da imunodeficiência adquirida) tenham ganhado uma conotação “do passado" para essas novas gerações, como se já não fossem tão perigosos nos dias atuais, transformando o avanço da doença em uma epidemia silenciosa (Pereira et al., 2012). Além disso, nota-se que a geração atual se expõe mais, haja vista que, devido ao advento da internet e, posteriormente, dos aplicativos de relacionamento, houve uma revolução sobre as formas de interação interpessoal, trazendo muitas facilidades, em especial para a população LGBTQIA+, que encontrou nestes instrumentos virtuais um espaço seguro de contato afetivo e sexual. No entanto, essa nova configuração de relacionamentos promoveu também a ideia de sexo casual com maior facilidade, o que se traduz em maiores taxas de risco na ausência de uma consciência de prevenção e cuidado (Nogueira et al., 2020).

Sob essa perspectiva, estudo de Pulga (2019) notou que análises a partir dos dados dos estudos demonstraram, em grande parte do espaço amostral, adolescentes que vivem em condições familiares e sociais desfavoráveis a um desenvolvimento sexual sadio, com estruturas familiares pouco estruturadas, geralmente com a falta de figura paterna, com 
casos constantes e repetitivos de gravidezes na adolescência, como demonstrado pelo instrumento de genograma familiar. No tocante ao aspecto social, há um perceptível hiato educacional, pois foi identificado, nas amostras de adolescentes que engravidaram, a evasão escolar, geralmente no ensino médio, a fim de buscar trabalho, geralmente informais e nas proximidades residenciais (Pinheiro et al., 2017).

Além disso, sob âmbito de educação sexual nas escolas, os resultados obtidos através de aplicação de formulários e escalas específicas, como a caracterização sociodemográfica dos adolescentes, idades, atitudes sexuais, permite a verificação do impacto do Programa de Educação Sexual na Turma (PEST), pois revelaram atitudes de maior concordância face às relações sexuais ocasionais, masturbação e relações sexuais sem o componente afetivo (Santos et al., 2010). Dessa forma, após esta participação, os adolescentes associaram mais facilmente o sexo com o prazer e funções corporais. Assim, os resultados parecem evidenciar uma mudança no sentido de atitudes face à sexualidade mais liberal, parecendo-se a mais desejável (Ribeiro et al., 2012).

Outros estudos apresentaram, contudo, resultados diferentes, em que as adolescentes revelaram posições mais liberais nas suas opiniões atitudinais face à sexualidade, através de uma escala que comportava temas como o amor, o afeto, a sexualidade pré-matrimonial, a virgindade, a masturbação, a concepção e a fidelidade, bem como uma atitude face à sexualidade mais positiva que os rapazes (Costa et al., 2013). Outrossim, demonstraram atitudes mais positivas nos adolescentes na subescala de práticas sexuais, referente às atitudes face ao planeamento familiar, à educação sexual e às práticas de sexo não convencional (Andrade et al., 2009). O aproveitamento escolar dos adolescentes influenciou, também, o impacto do PEST nas suas atitudes face à sexualidade, porque apenas os adolescentes sem retenções as alteraram significativamente, expressando uma maior concordância com as relações sexuais ocasionais, com a masturbação e com as relações sexuais sem componente afetiva. Assim, estes resultados parecem realçar a necessidade de estruturar intervenções que consigam ser o mais abrangentes possível e, por isso, não sejam condicionadas pelas competências escolares dos adolescentes (Flora et al., 2013).

De acordo com a Organização Mundial da Saúde, a sexualidade é uma necessidade básica e um componente do ser humano que não pode ser dissociado de outros aspectos da vida. Sexualidade é muito mais do que o coito e não se limita à presença ou não do orgasmo, é a energia que motiva o encontro para o amor, o contato e a intimidade, implicando em saúde física e mental (Moreira et al., 2021). Assim, se a saúde é um direito primordial, a saúde sexual também deve ser considerada um direito humano básico, que envolve o corpo, a história, as relações afetivas e a cultura dos homens. Apesar disso, ainda há relutância em problematizar esse tema abertamente, como parte da educação de crianças e jovens partindo ainda do ambiente familiar (Camargo et al., 2009).

Os resultados dos estudos de Almeida et al. (2017) contribuem para este repensar e exercem o papel de conscientização em uma população que cada vez mais apresenta histórico de ISTs/HIV, gestações precoces e frustrações impactantes no âmbito emocional. A idade média de iniciação sexual dos brasileiros situa-se na faixa dos 15 anos de idade, justificando a necessidade de ações de saúde enfáticas direcionadas à população adolescente e jovem. Os bate-papos educativos constituem a estratégia principal na abordagem desses assuntos, uma vez que se torna ferramenta de debate que, pela informalidade, permite que os alunos, bem como pais e professores, se expressem e se sintam à vontade para expor suas ideias e anseios (Sousa et al., 2018). Além disso, oficinas com a demonstração dos métodos contraceptivos existentes, permitindo que visualizem cada um deles, assim como a instrução da forma como devem ser utilizados, são recursos especialmente válidos para suscitar o interesse dos estudantes e assumir impacto maior no entendimento de sexo seguro e anticoncepção (Araújo et al., 2019). Tratar de temas atuais, normalmente estigmatizados e reprimidos anteriormente, possibilita a tomada de consciência acerca da sua existência, desmitificando tabus e contribuindo para a redução das 
desigualdades sociais e de gênero, do preconceito e da discriminação, bem como pode contribuir para a diminuição da vulnerabilidade aos jovens em relação às infecções sexualmente transmissíveis (Neves et al., 2012).

\section{Considerações Finais}

Diante desse cenário, é importante que jovens sejam orientados em relação às suas práticas sexuais. Para além do estudo sobre anatomia, a educação sexual proporciona o acesso a informações sobre diferentes aspectos da sexualidade. A partir dela, pessoas se tornam aptas a exercerem seus desejos sexuais e reprodutivos, estando informados quanto à sua saúde, haja vista que a sexualidade é costurada por diversas dimensões, como a biológica, social e cultural. Porém, nota-se que muitos educadores não se sentem seguros, apoiados ou capacitados para levar adiante projetos ou conteúdos sobre educação sexual. Além disso, a iniciação sexual de adolescentes que tiveram informação acontece mais tarde do que a de outros que foram impedidos de tê-la.

\section{Referências}

Almeida, R., et al. (2017). Conhecimento de adolescentes relacionados às doenças sexualmente transmissíveis e gravidez. Rev. Bras. Enferm., 70 (5), 10331039.

Andrade, H., et al. (2009). Mudanças no comportamento sexual seguindo programa de educação sexual em escolas públicas brasileiras. Cadernos de Saúde Pública, 25 (5), 1168-1176.

Araújo, F., et al. (2019). Caracterização Das Infecções Sexualmente Transmissíveis Em Usuários Da Atenção Básica: Uma Revisão Integrativa. Revista Uningá, 54 (2), 204-221.

Camargo, E., et al. (2009). Adolescentes: conhecimento sobre sexualidade antes e depois de participar da prevenção oficinas. Ciência e Saúde Coletiva, 14 (3), 937-946.

Costa, A., et al. (2013). Vulnerabilidade de adolescentes escolares às DST/HIV, em Imperatriz. Rev. Gaúcha Enferm., 34 (3), $179-186$.

Flora, M. C., et al. (2013). Intervenções de educação sexual em adolescentes: uma revisão sistemática da literatura. Revista de Enfermagem Referência, 3 (10), $125-134$.

Ministério da Saúde (Tabnet). Departamento de informática do SUS. (2017). Brasília, DF. <http://datasus.saude.gov.br/informacoes-de-saude>.

Moreira, G. B. C., et al. (2021). Adolescentes e as infecções sexualmente transmissíveis: comportamentos de risco e fatores contextuais que contribuem para o aumento da incidência no Brasil. Portal de Periódicos da Faculdade Ciências Médicas de Minas Gerais, 5 (1), 1-13.

Neves, R., et al. (2012). Simultaneidade de comportamentos de risco para infecções sexualmente transmissíveis em adolescentes brasileiros. Epidemiol Serv Saúde, 26 (5), 443-454.

Nogueira, F. J. S., et al. (2020). Educação sexual nas escolas: um desafio para os profissionais da saúde e educação. Revista Brasileira de Educação em Saúde, $10(3), 146-155$.

Pereira, C. S., et al. (2012). Projetos PET- Saúde e Educação para a Saúde: construindo saberes e práticas. Revista Brasileira de Educação Médica, 36 (1), $172-177$.

Pinheiro, A. S., et al. (2017). A estratégia Saúde na Família e a escola na educação sexual: uma perspectiva de intersetorialidade. Revista Brasileira de Educação em Saúde, 5 (3), 803-822.

Pulga, V. (2019). A importância da educação sexual nas escolas: uma possibilidade de expandir os conhecimentos adquiridos no curso de medicina para a comunidade. Fronteira Sul, Passo Fundo. 13 p.

Ribeiro, J. M., et al. (2012). Atitudes face à sexualidade nos adolescentes num programa de educação sexual. Revista Psicologia, Saúde e Doenças, 13 (2), $340-355$.

Santos, R. C., et al. (2010). Promoção da saúde sexual: desafios do Vale do São Francisco. Revista Psicologia \& Saúde, 22 (3), $499-506$.

Sousa, B., et al. (2018). Comportamento sexual e fatores associados em adolescentes da zona rural. Rev. Saúde Pública, 52 (39), 1-8. 